\title{
Prognostic value of visceral pleural invasion in resected non-small cell lung cancer diagnosed by using a jet stream of saline solution
}

\author{
Riichiroh Maruyama, MD \\ Fumihiro Shoji, MD \\ Tatsuro Okamoto, MD \\ Tetsuya Miyamoto, MD \\ Tetsuro Miyake, MD \\ Tomomi Nakamura, MD \\ Jiro Ikeda, MD \\ Hiroshi Asoh, MD \\ Masafumi Yamaguchi, MD \\ Ichiro Yoshino, MD \\ Yukito Ichinose, MD
}

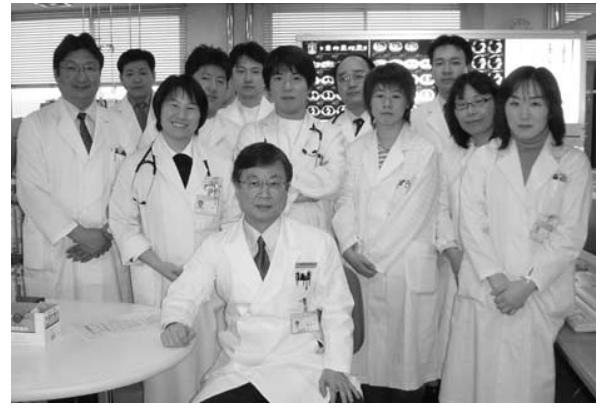

Front row, left to right: Asoh, Nakamura, Ichinose, Ikeda, Inoue, Oshima, Nishida; back row, left to right: Watanabe, Maruyama, Miyamoto, Shoji, Okamoto
Objective: Visceral pleural invasion caused by non-small cell lung cancer is a factor in the poor prognosis of patients with that disease. We investigated the relationship between the diagnosis of visceral pleural invasion by using a jet stream of saline solution, which was previously reported as a new cytologic method to more accurately detect the presence of visceral pleural invasion, and prognosis.

Methods: From January 1992 through December 1998, 143 consecutive patients with peripheral non-small cell lung cancer that appeared to reach the visceral pleura underwent a surgical resection at the Department of Thoracic Oncology, National Kyushu Cancer Center. The surface of the visceral pleura in patients undergoing lung cancer resection was irrigated with a jet stream of saline solution. The diagnosis of visceral pleural invasion was determined by means of either a pathologic examination or by means of a jet stream of saline solution. In addition, a cytologic examination of the pleural lavage fluid obtained immediately after a thoracotomy was evaluated.

Results: Forty-nine (34\%) resected tumors were identified as having visceral pleural invasion. The diagnosis of visceral pleural invasion in 31, 6, and 12 patients was determined by using a jet stream of saline solution alone, pathologic examination alone, or both, respectively. The visceral pleural invasion and positive findings of intrapleural lavage cytology were linked. Although there was no significant difference between the incidence of distant metastases in the patients with visceral pleural invasion and those without visceral pleural invasion, the incidence of local recurrence, especially regarding carcinomatous pleuritis (malignant pleural effusion, pleural dissemination, or both), in the patients with visceral pleural invasion was significantly higher than in those without visceral pleural invasion. The recurrence-free survival of patients with visceral pleural invasion was significantly shorter than that of patients without visceral pleural invasion $(P=.004)$, even patients with stage I disease $(P=.02)$. There was also a significant difference between the patients with or without visceral pleural invasion in the overall survival $(P=.02)$. Visceral pleural invasion was independently associated with a poor recurrence-free survival on the basis of multivariate analyses $(P=.03)$, as were sex $(P=.03)$, age $(P=002)$, and the stage of the disease $(P<.0001)$.

Conclusions: This study confirmed that the jet stream of saline solution method in addition to ordinary pathologic examination was useful for detecting visceral pleural invasion, which is considered to be one of the causes of local recurrence, especially in carcinomatous pleuritis. 


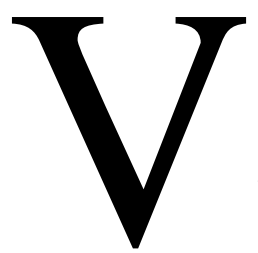

isceral pleural invasion (VPI) is a factor in the poor prognosis of patients with non-small cell lung cancer (NSCLC). ${ }^{1,2}$ The diagnosis of VPI is usually confirmed by means of a pathologic examination (PE) alone. PE is based on 1 or 2 cut slices of the resected tumor. Although PE can easily confirm VPI when the tumor is clearly visible on the visceral pleura, such cases are relatively rare. Therefore it remains unclear as to whether a tumor can be reliably considered to have no VPI on the basis of PE alone. To resolve this problem, we previously reported a simple method involving a cytologic examination of cells desquamated from the visceral pleura by using a jet stream of saline solution (JSS). This method is considered to be significantly more sensitive and accurate than ordinary PE in detecting VPI by lung cancer. ${ }^{3}$

We retrospectively investigated the relationship between a diagnosis of VPI in patients with resected NSCLC by using the JSS method and recurrent site and the prognosis.

\section{Methods}

\section{Patients and Methods}

From January 1992 through December 1998, 143 consecutive patients with peripheral NSCLC that appeared to reach the visceral pleura and that either did not adhere to or did not invade the surrounding tissue underwent a surgical resection at the Department of Thoracic Oncology, National Kyushu Cancer Center. This study included the 90 cases of a former report about JSS. ${ }^{3}$ Any patients with diffuse pleural adhesions, distant metastases, and T4 disease were excluded. The patients consisted of 81 men and 62 women. The median age of the patients was 64 years, with a range of 24 to 90 years. A complete surgical resection consisted of a lobectomy $(\mathrm{n}=132)$, bilobectomy $(\mathrm{n}=5)$, pneumonectomy $(\mathrm{n}=$ $3)$, or segmentectomy $(n=3)$. The JSS method was performed as previously described. ${ }^{3}$ Briefly, the surface of the visceral pleura in patients with resected lung cancer was irrigated twice with a jet stream of heparinized saline solution by using a $20-\mathrm{mL}$ syringe with a 21-gauge needle immediately after performing a surgical resection. The distance between the tip of the needle and the pleural surface was kept at approximately $2 \mathrm{~cm}$, and a total of 40 $\mathrm{mL}$ of saline solution containing cells desquamated from the visceral pleural surface was collected and then centrifuged at 1000 rpm for 10 minutes. Thereafter, the obtained sediment was stained by using the Giemsa and Papanicolaou method for cytologic examination. When it was necessary to distinguish cancer cells from reactive mesothelial cells, anticarcinoembryonic antigen staining, alcian blue staining, and periodic acid-Schiff reactions were performed. In addition, a cytologic examination of pleural lavage fluid obtained immediately after a thoracotomy was evaluated in all but 5 patients. The pathologic stage of the disease was based on the TNM classification of the Union Internationale Contre Cancer. ${ }^{4}$ The pathologic stage of the tumors in this series was IA in 49 patients, IB in 46 patients, IIA in 3 patients, IIB in 14 patients, IIIA in 28 patients, and IIIB in 3 patients. The histologic analysis of the tumor was based on the World Health Organization classification for cell types. ${ }^{5}$ One hundred sixteen patients had
TABLE 1. Rate of positive findings of VPI classified by means of JSS, PE, or either method according to the tumor stage

\begin{tabular}{|c|c|c|c|}
\hline \multirow[b]{2}{*}{ Pathologic stage } & \multicolumn{2}{|c|}{ No. of positive findings } & \multirow{2}{*}{$\begin{array}{c}\text { Total no. of } \\
\text { positive findings }\end{array}$} \\
\hline & JSS & PE & \\
\hline $\mathrm{IA}(\mathrm{n}=49)$ & $11(22 \%)$ & 一 & $11(22 \%)$ \\
\hline $\mathrm{IB}(\mathrm{n}=46)$ & $17(37 \%)$ & $9(20 \%)$ & $21(46 \%)$ \\
\hline $\mathrm{II}(\mathrm{n}=17)$ & $3(18 \%)$ & $3(18 \%)$ & $4(24 \%)$ \\
\hline $\mathrm{III}(\mathrm{n}=31)$ & $12(39 \%)$ & $6(19 \%)$ & $13(42 \%)$ \\
\hline Total $(n=143)$ & $43(30 \%)$ & $18(13 \%)$ & $49(34 \%)$ \\
\hline
\end{tabular}

$V P I$, Visceral pleural invasion; JSS, jet stream of saline solution; $P E$, pathologic examination.

TABLE 2. Rate of positive findings of VPI classified by means of JSS, PE, or either method according to $\mathbf{N}$ status

\begin{tabular}{lccc}
\hline & \multicolumn{2}{c}{$\begin{array}{c}\text { No. of positive } \\
\text { findings }\end{array}$} & $\begin{array}{c}\text { Total no. of } \\
\text { N status }\end{array}$ \\
\cline { 2 - 3 } & JSS & PEsitive findings \\
\hline $\mathrm{n} 0(\mathrm{n}=95)$ & $28(29 \%)$ & $9(9 \%)$ & $32(34 \%)$ \\
$\mathrm{n} 1(\mathrm{n}=17)$ & $3(18 \%)$ & $3(18 \%)$ & $4(24 \%)$ \\
$\mathrm{n} 2$ or more $(\mathrm{n}=31)$ & $12(39 \%)$ & $6(19 \%)$ & $13(42 \%)$ \\
\hline
\end{tabular}

$V P I$, Visceral pleural invasion; JSS, jet stream of saline solution; $P E$, pathologic examination.

TABLE 3. Relationship between pleural lavage cytologic findings and VPI as diagnosed with either diagnostic method

\begin{tabular}{lcc}
\hline & \multicolumn{2}{c}{ VPI } \\
\cline { 2 - 3 } Cytologic findings on intrapleural lavage & Present & Absent \\
\hline Positive $(n=13)$ & 13 & 0 \\
Negative $(n=125)$ & 33 & 92 \\
\hline
\end{tabular}

$V P I$, Visceral pleural invasion.

adenocarcinoma, 19 had squamous cell carcinoma, 6 had adenosquamous cell carcinoma, and 2 had large cell carcinoma. After the operation, the patients were re-examined once every 3 months for 5 years and thereafter at 6-month intervals. The evaluations included a physical examination and chest roentgenography at each visit and computed tomography of the chest, magnetic resonance imaging of the brain, and a bone scan every year.

\section{Statistical Analysis}

Statistical analyses were performed by using either $\chi^{2}$ analysis or the Fisher exact test for various clinicopathologic factors. The duration of the recurrence-free survival was calculated from the date of operation until either the first evidence of recurrence or death of any cause. Survival was calculated from the date of operation until death of any cause or the date of the last follow-up (censored). The recurrence-free interval and survival curves were determined by using the Kaplan-Meier method, and differences in 
TABLE 4. Incidence of recurrent disease in the patients with or without VPI as diagnosed with JSS, PE, or either method

\begin{tabular}{|c|c|c|c|c|c|c|}
\hline \multirow[b]{2}{*}{ Recurrent site } & \multicolumn{2}{|c|}{ JSS VPI } & \multicolumn{2}{|c|}{ PE VPI } & \multicolumn{2}{|c|}{ Either method VPI } \\
\hline & $\begin{array}{l}\text { Present } \\
(n=43)\end{array}$ & $\begin{array}{c}\text { Absent } \\
(n=100)\end{array}$ & $\begin{array}{l}\text { Present } \\
(\mathrm{n}=18) \\
\end{array}$ & $\begin{array}{c}\text { Absent } \\
(\mathrm{n}=125)\end{array}$ & $\begin{array}{l}\text { Present } \\
(\mathrm{n}=49) \\
\end{array}$ & $\begin{array}{c}\text { Absent } \\
(n=94)\end{array}$ \\
\hline Local recurrence & $12(28 \%)^{*}$ & $4(4 \%)$ & $4(22 \%)$ & $12(10 \%)$ & $12(24 \%) \S$ & $4(4 \%)$ \\
\hline $\begin{array}{l}\text { Carcinomatous } \\
\text { pleuritis }\end{array}$ & $7(16 \%) \dagger$ & 0 & $3(17 \%) \ddagger$ & $4(3 \%)$ & $7(14 \%) \|$ & 0 \\
\hline Other sites & $5(12 \%)$ & $4(4 \%)$ & $1(6 \%)$ & $8(6 \%)$ & $5(10 \%)$ & $4(4 \%)$ \\
\hline Distant metastases & $8(19 \%)$ & $26(26 \%)$ & $7(39 \%)$ & $27(22 \%)$ & $12(24 \%)$ & $22(23 \%)$ \\
\hline
\end{tabular}

$V P I$, Visceral pleural invasion; JSS, jet stream of saline solution; $P E$, pathologic examination.

${ }^{*} P=.0001$.

$\dagger P=.0002$.

$\ddagger P=.04$.

$\S P=.0003$.

$\| P=.0004$.

their distribution were evaluated by means of the log-rank test. ${ }^{6,7}$ The Cox proportional hazards models were applied for the multivariate analysis. $^{8}$ All data were analyzed with Abacus Concepts, Survival Tools for StatView (Abacus Concepts, Inc, Berkeley, Calif).

\section{Results}

Of the 143 tumors evaluated that were located peripherally and suspected of reaching the visceral pleura, $49(34 \%)$ were diagnosed to invade the visceral pleura by means of PE, the JSS method, or both. The diagnosis of VPI was made on the basis of the JSS method in 43 tumors and on the basis of PE in 18 tumors. Twelve tumors were found to have invaded the visceral pleura by means of both the JSS method and PE. The presence of VPI for the 49 resected tumors diagnosed by using the JSS method, PE, or both was considered to be true positive in this study. There were 6 false-negative results with the JSS method and 31 with PE. The sensitivity and accuracy of the JSS method for the diagnosis of VPI were $88 \%$ and $96 \%$, respectively. In contrast, the sensitivity and accuracy of PE were $37 \%$ and $78 \%$, respectively. The JSS method was significantly more sensitive and accurate than PE for the diagnosis of VPI. Table 1 shows the incidence of VPI as diagnosed with the JSS method and with PE according to pathologic stage. The presence of VPI for 11 (22\%) patients with stage IA disease was diagnosed with the JSS method. As shown in Table 2, there was no significant relationship between the extensive N2 involvement and VPI. VPI and a positive finding on intrapleural lavage after a thoracotomy was linked, as shown in Table 3 . No positive finding in the intrapleural lavage was found in our series in cases without VPI diagnosed on the basis of either the JSS method or PE. During a more than 5-year observation, we experienced 16 cases of local recurrence (metastases of hilar or mediastinal lymph nodes in 9 cases and carcinomatous pleuritis in 7 cases) and 34 cases of recurrence at distant organs. We defined malig- nant pleural effusion, pleural dissemination, or both as carcinomatous pleuritis in this study. Although there was no significant difference between the incidence of distant metastases in the patients with VPI and those without VPI, the incidence of local recurrence, especially regarding carcinomatous pleuritis, in the patients with VPI was significantly higher than in those without VPI, as shown in Table 4. Patients who otherwise were classified as having stage I disease had a significantly shorter recurrence-free survival if their VPI was present compared with that in patients with stage I disease without VPI, as shown in Figure $1, A(P=$ .02 ). All stages of patients with VPI had significantly shorter recurrence-free survivals than the patients without VPI, as shown in Figure $1, B(P=.004)$. As shown in Figure 2, there was also a significant difference between the patients with or without VPI in terms of overall survival ( $P$ $=.02$ ). In a multivariate analysis model that included sex, age, histologic type, pathologic stage, VPI, and positive cytologic finding on intrapleural lavage, VPI was an independent prognostic factor $(P=.03)$, as were sex $(P=.03)$, age $(P=.002)$, and the stage of the disease $(P<.0001$; Table 5).

\section{Discussion}

VPI is a factor of poor prognosis. ${ }^{1,2} \mathrm{PE}$ alone usually confirms the diagnosis of VPI. However, it remains questionable as to whether a tumor can be considered reliably to have no VPI on the basis of PE findings alone. We previously reported a simple method involving a cytologic examination of cells desquamated from the visceral pleura by using the JSS method, which was significantly more sensitive and accurate than ordinary PE in detecting VPI caused by lung cancer. ${ }^{3}$ This is a sequel report about JSS, which is a useful method to detect VPI. This study accumulated over 140 cases, and the follow-up period was sufficient to analyze the relationship between the presence of VPI detected 

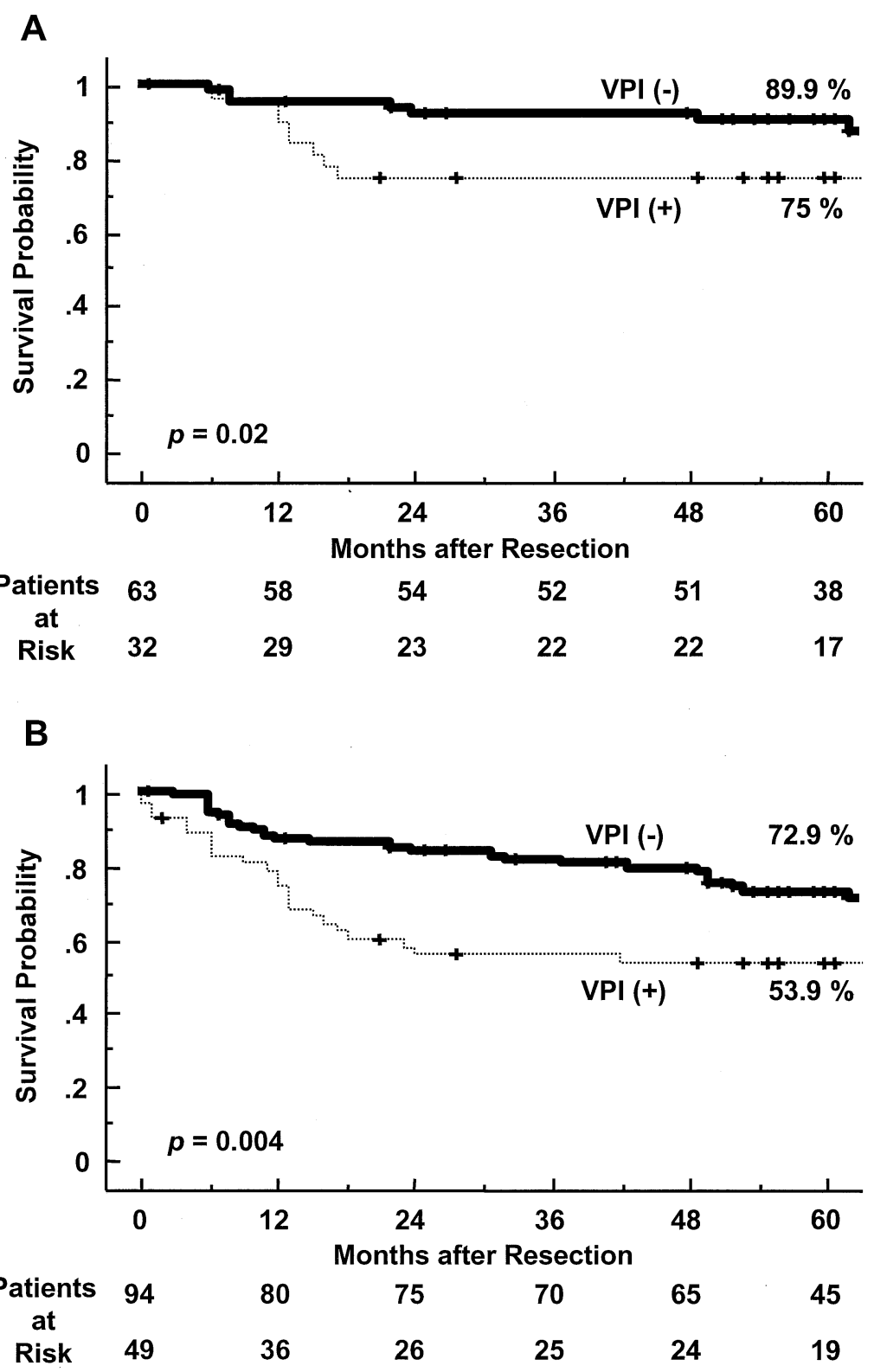

Figure 1. A, Recurrence-free survival in patients with stage I disease with or without VPI, as diagnosed by means of JSS or PE. B, Recurrence-free survival in all stages with or without VPI, as diagnosed by means of JSS or PE.

by means of our method and the prognosis. Bunker and associates ${ }^{9}$ reported that an evaluation of VPI by means of hematoxylin and eosin examination alone might be indeterminate, and Verhoeff-Van Gieson elastic stains can be helpful in the diagnosis of VPI. However, there are limitations in making a diagnosis of VPI on the basis of PE alone because the diagnosis of VPI by PE usually is based on 1 or 2 cut slices of the resected tumor. Our findings reconfirmed that the JSS method is significantly more sensitive and accurate than ordinary PE in detecting VPI. The methodology for the JSS after resection for tumor close to the pleura is simple to complete and thus could affect the ultimate staging of patients. Unfortunately, the results are dependent on the ability of the cytologic team, which might not be universally available. Recently, the Cancer and Leukemia Group B trial demonstrated micrometastatic tumor cells in the lymph nodes of patients with stage I NSCLC by using standard and quantitative real-time reverse transcriptase-polymerase chain reaction for carcinoembryonic antigen. ${ }^{10}$ Newer molecular techniques like this also might increase the yield of positivity. After our report on the usefulness of the JSS method, Saito and colleagues ${ }^{11}$ reported on the diagnosis of 


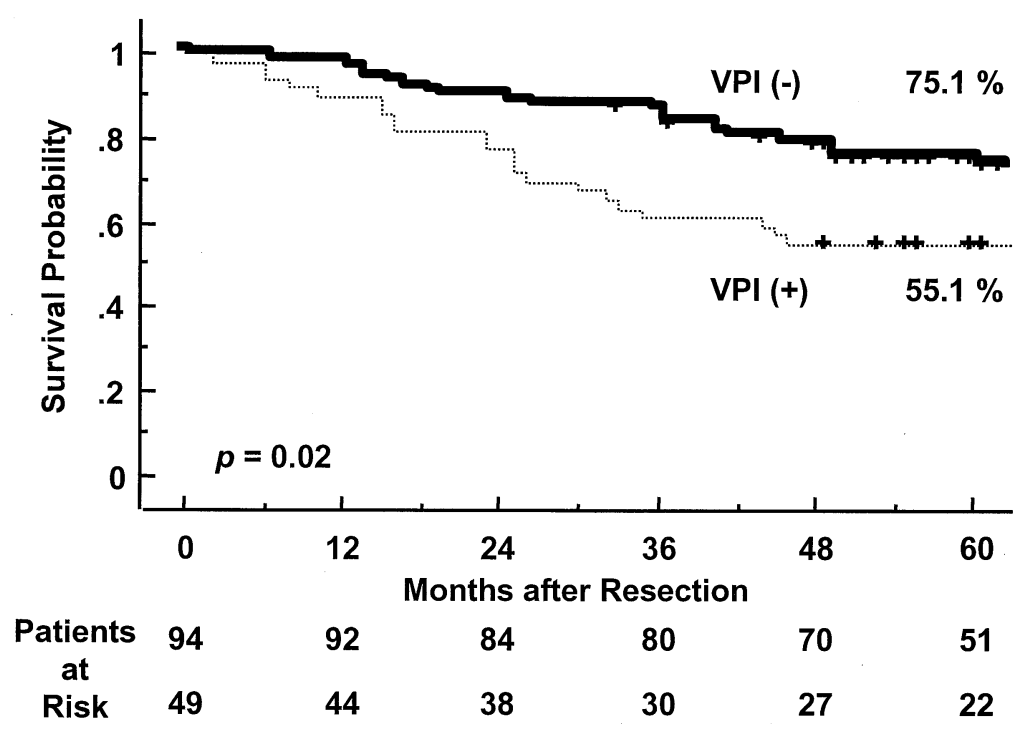

Figure 2. Overall survival with or without VPI, as diagnosed by means of JSS or PE.

VPI by using intraoperative touch cytology. Their method was simple and useful for detecting VPI; however, their follow-up was too short to fully analyze the prognosis. Manac' $h$ and coworkers ${ }^{2}$ reported that VPI was associated with a higher frequency of $\mathrm{N} 2$ involvement, and cancerrelated death was mainly caused by distant metastases rather than local recurrence. Their results support the hypothesis that exfoliated tumor cells are drained through the pleural lymphatics to the mediastinal lymphatic pathways and then into the bloodstream. There was no relationship between the findings of VPI and the N status in our series. The prognosis of patients with positive cytologic findings on intrapleural lavage was poor compared with that in patients with negative findings. ${ }^{12,13}$ Therefore they suggested that patients with positive cytologic findings on intrapleural lavage were considered to be in a prestage of carcinomatous pleuritis and thus should be upstaged. It was important that positive cytologic findings on intrapleural lavage were never present in cases without VPI in our series. Ichinose and associates ${ }^{14}$ reported that intraoperative intrapleural hypotonic cisplatin treatment was found to effectively suppress the appearance of malignant pleural effusion, pleural dissemination, or both in selected patients who demonstrated positive pleural lavage cytology findings.

Our JSS method in addition to ordinary PE is therefore useful in detecting VPI, which is considered to be one of the causes of local recurrence, especially in patients with carcinomatous pleuritis. VPI is therefore considered to be an important prognostic factor and index for better selecting patients who can most benefit from adjuvant therapy consisting of hypotonic cisplatin treatment.
TABLE 5. Multivariate findings of the recurrence-free survival

\begin{tabular}{|c|c|c|c|}
\hline Variable & Hazard ratio & $\begin{array}{c}95 \% \\
\text { Confidence } \\
\text { interval }\end{array}$ & $P$ value \\
\hline Sex: female/male & 1.96 & $1.08-3.57$ & .03 \\
\hline $\begin{array}{l}\text { Age }^{*} \text { : Elderly }(>64 \mathrm{y}) / \\
\text { younger }(<64 \mathrm{y})\end{array}$ & 2.76 & $1.45-5.24$ & .002 \\
\hline $\begin{array}{l}\text { Histologic type: } \\
\text { nonsquamous/squamous }\end{array}$ & 1.44 & $0.49-4.21$ & .51 \\
\hline \multicolumn{4}{|l|}{ Pathologic staget: } \\
\hline $\mathrm{IB} / \mathrm{IA}$ & 3.69 & $1.19-11.5$ & .02 \\
\hline II/IA & 7.65 & $2.26-25.9$ & .001 \\
\hline III/IA & 22.0 & $7.36-65.7$ & $<.0001$ \\
\hline VPI & 2.15 & $1.06-4.33$ & .03 \\
\hline $\begin{array}{l}\text { Positive cytologic finding } \\
\text { on intrapleural lavage }\end{array}$ & 1.95 & $0.79-4.83$ & .15 \\
\hline
\end{tabular}

$V P I$, Visceral pleural invasion.

*The number of elderly and younger patients was 71 and 72 , respectively. †The pathologic stage of the tumors was IA in 49 patients, IB in 46 patients, II in 17 patients, and III in 31 patients.

We thank Dr Brian T. Quinn for critical comments on the manuscript and Miss Yumiko Oshima for reviewing the patient chart.

\section{References}

1. Ichinose Y, Yano T, Asoh H, et al. Prognostic factors obtained by a pathologic examination in completely resected non-small-cell lung cancer. An analysis in each pathologic stage. $J$ Thorac Cardiovasc Surg. 1995;110(3):601-5.

2. Manac'h D, Riquet M, Medioni J, et al. Visceral pleura invasion by 
non-small cell lung cancer: an underrated bad prognostic factor. Ann Thorac Surg. 2001;71(4):1088-93.

3. Ichinose $\mathrm{Y}$, Yano $\mathrm{T}$, Asoh $\mathrm{H}$, et al. Diagnosis of visceral pleural invasion in resected lung cancer using a jet stream of saline solution. Ann Thorac Surg. 1997;64(6):1626-9.

4. Sobin LH, Wittenkind C. TNM classification of malignant tumors. New York: Wiley-Liss; 1997.

5. Travis WD, Corrin B, Shimosato Y, et al. International histological classification of tumors. Histological typing of lung and pleural tumors. World Health Organization. 3rd ed. Berlin: Springer Verlag; 1999.

6. Kaplan E, Meier P. Nonparametric estimation from incomplete observation. J Am Stat Assoc. 1958;53:457-81.

7. Mantel N. Evaluation of survival data and two new rank order statistics arising in its consideration. Cancer Chemother Rep. 1966;50(3):163-70.

8. Cox DR. Regression models and life tables. J R Stat Soc. 1972;34: $187-220$

9. Bunker ML, Raab SS, Landreneau RJ, Silverman JF. The diagnosis and significance of visceral pleural invasion in lung carcinoma. Histologic predictors and the role of elastic stains. Am J Clin Pathol. 1999;112(6):777-83.
10. D'Cunha J, Corfits AL, Herndon JE 2nd, et al. Molecular staging of lung cancer: real-time polymerase chain reaction estimation of lymph node micrometastatic tumor cell burden in stage I non-small cell lung cancer-preliminary results of Cancer and Leukemia Group B Trial 9761. J Thorac Cardiovasc Surg. 2002;123(3):484-91.

11. Saito Y, Yamakawa Y, Kiriyama M, et al. Diagnosis of visceral pleural invasion by lung cancer using intraoperative touch cytology. Ann Thorac Surg. 2002;73(5):1552-7.

12. Dresler CM, Fratelli C, Babb J. Prognostic value of positive pleural lavage in patients with lung cancer resection. Ann Thorac Surg. 1999; 67(5):1435-9.

13. Ichinose Y, Tsuchiya R, Yasumitsu T, et al. Prognosis of non-small cell lung cancer patients with positive pleural lavage cytology after a thoracotomy: results of the survey conducted by the Japan Clinical Oncology Group. Lung Cancer. 2001;31(1):37-41.

14. Ichinose $\mathrm{Y}$, Tsuchiya R, Koike T, et al. A prematurely terminated phase III trial of intraoperative intrapleural hypotonic cisplatin treatment in patients with resected non-small cell lung cancer with positive pleural lavage cytology: the incidence of carcinomatous pleuritis after surgical intervention. J Thorac Cardiovasc Surg. 2002;123(4):695-9.

\section{JTCVS On-Line Manuscript Submission and Review}

\section{Please visit http://www.editorialmanager.com/jtcvs/}

Effective September 15, 2001, authors and reviewers may submit manuscripts and reviews electronically via Editorial Manager, our new Web-based system with full electronic submission, review, and status update capabilities.

As we move from paper to electronic submissions, the Editorial Office will make proxy submissions of all manuscripts accompanied by a diskette containing the electronic files of the text, tables, and figures. Editors, authors, and reviewers will receive automatic e-mails when significant events occur.

We strongly encourage all authors and reviewers to use Editorial Manager. Although we will continue to accommodate the submission of paper manuscripts for some months, our goal is to be completely electronic within 9 to 12 months.

All individuals currently in our database for whom we have e-mail addresses will receive via e-mail a system-assigned username and password that can be used to log in to the system without prior registration. All those not receiving the e-mail must register the first time they use the system.

As with any broad systemic change, the conversion to the new system will take some time to complete. We ask your patience as we replace our in-office database with the new system. We also encourage you to take advantage of the speed and efficiency that the new system will provide for us all: editor, author, reviewer, and publisher. 